\title{
A Detailed Study of the Role of Fermi Energy in Determining Properties of Superconducting NbN
}

\author{
G. P. Malik ${ }^{1,2}$ \\ ${ }^{1}$ School of Environmental Sciences, Jawaharlal Nehru University, New Delhi, India \\ ${ }^{2}$ Present Address: B 208 Sushant Lok 1, Gurgaon, Haryana, India \\ Email: gulshanpmalik@yahoo.com,malik@mail.jnu.ac.in
}

How to cite this paper: Malik, G.P. (2017) A Detailed Study of the Role of Fermi Energy in Determining Properties of Superconducting NbN. Journal of Modern Physics, 8, 99-109.

http://dx.doi.org/10.4236/jmp.2017.81009

Received: December 27, 2016

Accepted: January 20, 2017

Published: January 23, 2017

Copyright $\odot 2017$ by author and Scientific Research Publishing Inc. This work is licensed under the Creative Commons Attribution International License (CC BY 4.0).

http://creativecommons.org/licenses/by/4.0/

c) (i) Open Access

\begin{abstract}
The recent concern with the role of Fermi energy $\left(E_{F}\right)$ as a determinant of the properties of a superconductor (SC) led us to present new $E_{F}$-dependent equations for the effective mass $\left(m^{*}\right)$ of superconducting electrons, their critical velocity, number density, and critical current density, and also the results of the calculations of these parameters for six SCs the $T_{c} s$ of which vary between 3.72 and $110 \mathrm{~K}$. While this work was based on, besides an idea due to Pines, equations for $T_{c}$ and the gap at $T=0$ that are explicitly $E_{F}$-dependent, it employed an equation for the dimensionless construct $y=k \theta \sqrt{2 m^{*}} / P_{0} \sqrt{E_{F}}$ that depends on $E_{F}$ only implicitly; $k$ in this equation is the Boltzmann constant, $\theta$ is the Debye temperature, and $P_{0}$ is the critical momentum of Cooper pairs. To meet the demand of consistency, we give here derivation of an equation for $y$ that is also explicitly $E_{F}$-dependent. The resulting framework is employed to (a) review the previous results for the six SCs noted above and (b) carry out a study of NbN which is the simplest composite SC that can shed further light on our approach. The study of NbN is woven around the primary data of Semenov et al. For the additional required inputs, we appeal to the empirical data of Roedhammer et al. and of Antonova et al.
\end{abstract}

\section{Keywords}

$E_{F}$-Incorporated Equations for $T_{\mathcal{C}} \Delta_{0}$, and $j_{0}$ of a Superconductor, $\mathrm{NbN}$

\section{Introduction}

Some of the recent studies [1]-[7] concerned with high- $T_{c}$ superconductors (SCs) have been motivated by the belief that Fermi energy $\left(E_{F}\right)$ plays an important role in determining their $T_{c} s$ and gap-structures. These studies make it natural to ask: why not incorporate $E_{F}$ (equivalently, chemical potential $\mu$ ) into the equations for the $T_{c}$ and the gap $\Delta$ of an SC, and then treat it as an independent variable? This is a departure from the usual practice because these parameters are conventionally calculated via equations 
sans $E_{F}$ because of the assumption

$$
E_{F} / k \theta \gg 1
$$

where $k$ is the Boltzmann constant and $\theta$ is the Debye temperature.

The proposed approach requires, besides the values of $T_{c}$ and $\Delta$, another property of the SC in order to determine $E_{F}$. Upon choosing critical current density $j_{0}$ of the SC, new equations for both elemental and composite SCs valid at $T=0$ were recently presented in [8] for $j_{0}$ and the following of their properties: $m^{*}, v_{0}$, and $n_{s}$, which denote, respectively, the effective mass of superconducting electrons, their critical velocity at which $\Delta_{0}$ vanishes, and the density of superconducting electrons. While the results of such a study for $\mathrm{Sn}, \mathrm{Pb}, \mathrm{MgB}_{2}$, YBCO, Bi-2212, and Tl-2212 were also reported in [8], it was based on, unlike the equations for $\Delta_{0}$ and $T_{c}$, an equation for the dimensionless construct $y$, defined below, that is dependent on $E_{F}$ only implicitly.

$$
y=\left(k \theta / P_{0}\right) \sqrt{2 m * / E_{F}},
$$

where $m^{*}, P_{0}$, and $E_{F}$ are in units of electron volts.

To meet the demand of consistency, we present here the derivation of a new equation for $y$ that also contains $E_{F}$ explicitly-to put it on par with the equations for $T_{c}$ and $\Delta_{0}$. While this leads us to review our earlier results, we also undertake here a detailed study of the superconducting properties of $\mathrm{NbN}$ because:

(i) It is the simplest composite SC different samples of which (a) have been fabricated by the same method of preparation, (b) are geometrically similar, but (c) differ in size (e.g., film thickness), and for which (d) data in the form $\left\{T_{c}, j_{0}, n_{e}\right\}$ are available, where $n_{e}$ is the density of conduction electrons. This is unlike the composite SCs dealt with earlier, which were not necessarily fabricated by the same method of preparation and for which the values of $j_{0}$ and $n_{e}$ were not available. We were then led to estimate the values of $j_{0}$ for these SCs from the data at $T=4.2 \mathrm{~K}$. Given the values of $T_{c}$ and $n_{e}$ for $\mathrm{NbN}$, we can now also shed light on the ratio $n_{s} / n_{e}$ as a function of $T_{c}$.

(ii) Since the value of the highest $T_{c}$ reported for it in [9] is $15.25 \mathrm{~K}$, it is the simplest composite SC for which we believe one-phonon exchange mechanism (OPEM) to be operative. This is unlike, e.g., $\mathrm{MgB}_{2}$ for which, given its $T_{c}$, we need to invoke the twophonon exchange mechanism (TPEM).

(iii) The above features make $\mathrm{NbN}$ the simplest testing ground for some key steps of our approach, such as the procedure followed for resolving $\theta_{\mathrm{NbN}}$ into $\theta_{\mathrm{Nb}}$ and $\theta_{\mathrm{N}}$.

The paper is organized as follows. In Section 2 are reproduced from [8] those equations that constitute our framework in the OPEM scenario, which may be defined as one in which the $T_{c}$ of an SC can be accounted for by a value of the interaction parameter $\lambda$ that satisfies the Bogoliubov constraint, i.e., $\lambda<0.5$. Section 3 is devoted to derivation of the new equation for $y$. The study of $\mathrm{NbN}$ is taken up in Section 4. A review of our earlier results is taken up in Section 5. The final two sections are devoted to a discussion and conclusions, respectively.

\section{2. $E_{F}$-Incorporated Equations for Various Properties of an SC}

Recalled below from [8] are some of the equations that we need for NbN. In these equations $\left|W_{0}\right|$ is to be identified with $\Delta_{0}$. Further, the equations have been written by 
assuming that $\mu, E_{P}$ and $\lambda$ have the same values at $T=0$ and $T=T_{\mathcal{c}}$, which is in accord with a tenet of the BCS theory. In the following we use $\mu$ and $E_{F}$ interchangeably because they will be seen to differ negligibly. The modified equation for $y$ will be derived in the next section.

Equation for $\left|W_{0}\right|$ :

$$
\frac{\lambda}{2} I_{1}\left(\mu,\left|W_{0}\right|\right)-\left[\frac{3}{4} I_{2}\left(\mu,\left|W_{0}\right|\right)\right]^{1 / 3}=0
$$

where

$$
I_{1}\left(\mu,\left|W_{0}\right|\right)=\int_{-k \theta}^{k \theta} \mathrm{d} \xi \sqrt{\xi+\mu} /\left(|\xi|+\left|W_{0}\right| / 2\right)
$$

and

$$
I_{2}\left(\mu,\left|W_{0}\right|\right)=\frac{4}{3}(\mu-k \theta)^{3 / 2}+\int_{-k \theta}^{k \theta} \mathrm{d} \xi \sqrt{\xi+\mu}\left[1-\xi / \sqrt{\left(\xi^{2}+W_{0}^{2}\right)}\right] .
$$

Equation for $T_{\vec{c}}$

$$
\frac{\lambda}{2} I_{3}\left(\mu, T_{c}\right)-\left[\frac{3}{4} I_{4}\left(\mu, T_{c}\right)\right]^{1 / 3}=0
$$

where

$$
I_{3}\left(\mu, T_{c}\right)=\int_{-k \theta}^{k \theta} \mathrm{d} \xi\left[\sqrt{\xi+\mu} \tanh \left(\xi / 2 k T_{c}\right)\right] / \xi
$$

and

$$
I_{4}\left(\mu, T_{c}\right)=\int_{-\mu_{1}}^{k \theta} \mathrm{d} \xi \sqrt{\xi+\mu}\left[1-\tanh \left(\xi / 2 k T_{c}\right)\right] .
$$

In the above equations

$$
\lambda \equiv[N(0) V]=\left[\left(1 / 4 \pi^{2}\right)\left(2 m^{*}\right)^{3 / 2} E_{F}^{1 / 2}\right] V, \quad(\hbar=1)
$$

After $\lambda$ has been determined via (7) with the input of $\theta, T_{c}$, and any assumed value of $\mu$, the corresponding value of $E_{F}$ can be determined by the following equation

$$
E_{F}=\left[(\lambda / 2) I_{3}\left(\mu, T_{c}\right)\right]^{2} \text {. }
$$

Equation for $y$ :

$$
1-\lambda[y \ln \{y /(y-1)\}+\ln (y-1)]=0
$$

This equation has been obtained by assuming that

$$
E_{3} / E_{1}, E_{2} / E_{1} \ll 1
$$

where

$$
E_{1}=k \theta, E_{2}=P_{0} \sqrt{E_{F} / 2 m^{*}}, E_{3}=P_{0}^{2} / 8 m^{*}\left(y=E_{1} / E_{2}\right) .
$$

Equation for $j_{0}\left(E_{F}\right)$ :

$$
j_{0}\left(E_{F}\right)=n_{s}\left(E_{F}\right) e^{*} v_{0}\left(E_{F}\right)=A_{5}(\theta / y)\left(\gamma / v_{g}\right)^{2 / 3} E_{F}^{2 / 3},\left(e^{*}=2 e\right)
$$

where 


$$
A_{5}=e /\left(6^{1 / 3} \pi^{2} k^{1 / 3} \hbar\right) \simeq 3.703 \times 10^{-4} \mathrm{CeV}^{-4 / 3} \cdot \mathrm{K}^{1 / 3} \cdot \mathrm{sec}^{-1} .
$$

\section{The Modified Equation for $y$ in the OPEM Scenario}

Equation (11) has been derived in [10] (pp. 115-120) by assuming Inequality (1). In order to do away with this inequality, we begin here with the following equation for moving CPs because the present derivation differs from the earlier one only beyond it.

$$
1=\left(V / 16 \pi^{3}\right) \int_{L}^{U} \mathrm{~d}^{3} p[\tanh (\beta C(p) / 2)+\tanh (\beta D(p) / 2)] /[C(p)+D(p)] .
$$

In this equation

$$
\begin{gathered}
L=-k \theta+P \alpha x-P^{2} / 8 m^{*} \\
U=k \theta-P \alpha x-P^{2} / 8 m^{*} \\
\alpha=\sqrt{E_{F} / 2 m^{*}}, \quad x=\cos (P, p), \quad \beta=1 / k T \\
C(p)=E_{F}+W / 2-P^{2} / 8 m^{*}-P \alpha x-p^{2} / 2 m^{*} \\
D(p)=E_{F}+W / 2-P^{2} / 8 m^{*}+P \alpha x-p^{2} / 2 m^{*}
\end{gathered}
$$

Equation (16) was obtained via a Bethe-Salpeter equation. It seems interesting to point out that when $P=0$, it reduces to the well known criterion of superconductivity derived by Thouless via the t-matrix approach, as can be seen from [11] and, in greater detail, in [12].

The equation for the critical momentum $P_{c}(T)$ at any temperature follows from (16) by putting $W=0$. In terms of $\xi=p^{2} / 2 m-E_{F}$, we then have

$$
1=(\lambda / 4) \int_{0}^{1} \mathrm{~d} x\left[I_{1}(x)+I_{2}(x)\right],
$$

where

$$
\begin{gathered}
I_{1}(x)=\int_{-E_{1}+E_{2} x}^{E_{1}-E_{2} x} \mathrm{~d} \xi \varphi(\xi) \tanh \left[(\beta / 2)\left(\xi+E_{2} x\right)\right] \\
I_{2}(x)=\int_{-E_{1}+E_{2} x}^{E_{1}-E_{2} x} \mathrm{~d} \xi \varphi(\xi) \tanh \left[(\beta / 2)\left(\xi-E_{2} x\right)\right], \\
\varphi(\xi)=\sqrt{1+\xi / E_{F}} /\left(\xi+E_{3}\right),
\end{gathered}
$$

and we have used (9), (13) and (19). Besides, justification to follow, we have dropped $E_{3}$ everywhere except in the denominator of (25) in order to avoid the singularity at $\xi=0$. Compared with the earlier equation for $y$, the new feature of (22) is that it has the additional factor of $\sqrt{1+\xi / E_{F}}$ in each of its constituents.

In order to obtain the $T=0(\beta=\infty)$ version of (22), we split both $I_{1}(x)$ and $I_{2}(x)$ into two parts: $I_{1}(x)$ into $I_{11}(x)$ and $I_{12}(x)$ for which the limits of integration are $\left(-E_{1}+E_{2} x\right)$ to $-E_{2} x$ and $-E_{2} x$ to $\left(E_{1}-E_{2} x\right)$, respectively, and $I_{2}(x)$ into $I_{21}(x)$ and $I_{22}(x)$, where the former is integrated from $\left(-E_{1}+E_{2} x\right)$ to $E_{2} x$ and the latter from $E_{2} x$ to $\left(E_{1}-E_{2} x\right)$. It is then seen that, when $T=0, \tanh (\cdots)=(-1)$ for $I_{11}(x)$ and $I_{21}(x)$ and $(+1)$ for the remaining parts.

Because the constituents of both $I_{1}(x)$ and $I_{2}(x)$ differ from one another only in the matter of limits and an overall sign, we now consider the following indefinite integral: 


$$
J=\int \mathrm{d} \xi \varphi(\xi)=\int \mathrm{d} z \sqrt{1+z} /\left(z+E_{3}^{\prime}\right),
$$

where we have used (25), put $z=\xi / E_{F}$ and $E_{3}^{\prime}=E_{3} / E_{F}$, whence

$$
J=2 \sqrt{z+1}-2 \sqrt{E_{3}^{\prime}-1} \arctan \left[\sqrt{(z+1) /\left(E_{3}^{\prime}-1\right)}\right] .
$$

Therefore, for $E_{3}^{\prime} \ll 1$ (as will be seen to be so), we obtain

$$
J=2 \sqrt{z+1}+2 i \arctan (i \sqrt{z+1})=2 \sqrt{z+1}-\ln [(1+\sqrt{z+1}) /(1-\sqrt{z+1})] .
$$

Taking into account the overall sign of $I_{11}(x),(28)$ yields

$$
\begin{aligned}
I_{11}(x)= & {\left[-2 \sqrt{1-E_{2}^{\prime} x}+2 \sqrt{1-E_{1}^{\prime}+E_{2}^{\prime} x}\right] } \\
& +\ln \left[\left\{\left(1+\sqrt{1-E_{2}^{\prime} x}\right) /\left(1-\sqrt{1-E_{2}^{\prime} x}\right)\right\}\left\{\left(1-\sqrt{1-E_{1}^{\prime}+E_{2}^{\prime} x}\right) /\left(1+\sqrt{1-E_{1}^{\prime}+E_{2}^{\prime} x}\right)\right\}\right]
\end{aligned}
$$

where $E_{i}^{\prime}=E_{i} / E_{F}(i=1,2)$. Since $E_{1}^{\prime} / E_{2}^{\prime}=E_{1} / E_{2}=y$, we replace $E_{2}^{\prime}$ in the above equation by $E_{1}^{\prime} / y$ in order to make contact with (11). $I_{12}(x), I_{21}(x)$ and $I_{22}(x)$ can be similarly calculated. For the sake of compactness, we define

$$
\begin{gathered}
u_{1}\left(E_{1}^{\prime}, x, y\right)=\sqrt{1-E_{1}^{\prime} x / y}, u_{2}\left(E_{1}^{\prime}, x, y\right)=\sqrt{1+E_{1}^{\prime} x / y} \\
u_{3}\left(E_{1}^{\prime}, x, y\right)=\sqrt{1-E_{1}^{\prime}(1-x / y)}, u_{4}\left(E_{1}^{\prime}, x, y\right)=\sqrt{1+E_{1}^{\prime}(1-x / y)} .
\end{gathered}
$$

Then substituting $I_{1}(x)$ and $I_{2}(x)$ into (22), we obtain

$$
1=(\lambda / 4) \int_{0}^{1} \mathrm{~d} x\left[T_{1}\left(E_{1}^{\prime}, x, y\right)+T_{2}\left(E_{1}^{\prime}, x, y\right)\right] \equiv T\left(\lambda, E_{1}^{\prime}, y\right)
$$

where

$$
\begin{gathered}
T_{1}\left(E_{1}^{\prime}, x, y\right)=4\left\{-u_{1}\left(E_{1}^{\prime}, x, y\right)-u_{2}\left(E_{1}^{\prime}, x, y\right)+u_{3}\left(E_{1}^{\prime}, x, y\right)+u_{4}\left(E_{1}^{\prime}, x, y\right)\right\}, \\
T_{2}\left(E_{1}^{\prime}, x, y\right)=2 \ln \left\{\frac{1+u_{1}\left(E_{1}^{\prime}, x, y\right)}{1-u_{1}\left(E_{1}^{\prime}, x, y\right)} \frac{1+u_{2}\left(E_{1}^{\prime}, x, y\right)}{1-u_{2}\left(E_{1}^{\prime}, x, y\right)} \frac{1-u_{3}\left(E_{1}^{\prime}, x, y\right)}{1+u_{3}\left(E_{1}^{\prime}, x, y\right)} \frac{1-u_{4}\left(E_{1}^{\prime}, x, y\right)}{1+u_{4}\left(E_{1}^{\prime}, x, y\right)}\right\},
\end{gathered}
$$

and $T\left(\lambda, E_{1}^{\prime}, y\right)$ has been defined for later convenience. Obtained by retaining the factor $\sqrt{1+\xi / E_{F}}$ in $I_{1}(x)$ and $I_{2}(x)$, (32) for $y$ is the equation we had set out to obtain. It generalizes (11) which was obtained without this factor. While we could earlier solve (11) in the OPEM scenario with the input of $\lambda$ alone, solution of (32) requires the additional input of $\theta$ and $E_{F}$. In order to carry out a quick consistency check of (32), we recall that upon solving (6) for $\operatorname{Sn}\left(\theta=195 \mathrm{~K}, T_{c}=3.72 \mathrm{~K}, \mu / k \theta=100\right)$, we had earlier obtained $\lambda=0.2466$. The solution of (11) then led to $y=21.726$. This is precisely the value we now obtain by solving (32) with the same inputs for $\lambda, \theta$, and $E_{1}^{\prime}=E_{1} / E_{F}=1 / 100$.

\section{Study of $\mathrm{NbN}$ Based on $E_{F}$-Incorporated Equations}

\subsection{Outline of Procedure}

Working in the OPEM scenario, we

(A) Solve. (6) with the input of $\theta$ and $T_{c}$ to determine $\lambda$ for different assumed values of $\mu$.

(B) Solve (32) to obtain the values of $y$ corresponding to each pair of $(\mu, \lambda)$ values obtained above. 
(C) Calculate $j_{0}$ via (14) for each triplet of $\{\mu, \lambda, y\}$ values till it is found to agree with its experimental value.

As predictions, this process also yields the values of $m^{\star}, n_{s}$, and $v_{0}$ via equations derived in [8] and noted in Table 3. As a further check, we calculate $\left|W_{0}\right|$ via (3) by employing the values of $\mu$ and $\lambda$ that led in (C) to the experimental value of $j_{0}$.

Before we can proceed as above, we need to fix the Debye temperature of the ions that cause pairing in $\mathrm{NbN}$, i.e., $\theta_{\mathrm{Nb}}$.

\subsection{Debye Temperature of $\mathrm{Nb}$ Ions in $\mathrm{NbN}$}

$\theta_{\mathrm{NbN}}$ is not quoted in [9]. The reported values for it vary in the range $250-335 \mathrm{~K}$ [13] [14] [15] [16]. We begin by adopting [13]

$$
\theta_{\mathrm{NbN}}=335 \mathrm{~K} \text {. }
$$

We now need to resolve $\theta_{\mathrm{NbN}}$ into $\theta_{\mathrm{Nb}}$ and $\theta_{\mathrm{N}}$, which must be different because masses of $\mathrm{Nb}$ and $\mathrm{N}$ ions are different. As in [8], we do so via the following equations

$$
\begin{gathered}
\theta_{\mathrm{NbN}}=0.5 \theta_{\mathrm{Nb}}+0.5 \theta_{\mathrm{N}} \\
\theta_{\mathrm{Nb}} / \theta_{\mathrm{N}}=\left[\left\{1+\sqrt{m_{\mathrm{N}} /\left(m_{\mathrm{N}}+m_{\mathrm{Nb}}\right)}\right\} /\left\{1-\sqrt{m_{\mathrm{N}} /\left(m_{\mathrm{N}}+m_{\mathrm{Nb}}\right)}\right\}\right]^{1 / 2},
\end{gathered}
$$

where $m_{\mathrm{N}}\left(m_{\mathrm{Nb}}\right)$ is the atomic mass of $\mathrm{N}(\mathrm{Nb})$. While the first of the above equations has been routinely used for binaries, the second equation has been derived [10] by assuming that the constituents of the binary simulate weakly coupled oscillations of a double pendulum. The equations above have been written by assuming that $\mathrm{Nb}$ is the upper bob of the double pendulum. With $m_{\mathrm{Nb}}=92.91, m_{\mathrm{N}}=14.007$, and $\theta_{\mathrm{NbN}}$ as in (33), the solutions of these equations yield

$$
\begin{aligned}
& \theta_{\mathrm{Nb}}=397.8 \mathrm{~K} \text { ( } \mathrm{Nb} \text { as the upper bob) } \\
& \theta_{\mathrm{Nb}}=105.7 \mathrm{~K} \text { ( } \mathrm{Nb} \text { as the lower bob), }
\end{aligned}
$$

the corresponding values for $\theta_{\mathrm{N}}$ being 272.2 and $564.3 \mathrm{~K}$ (which we do not need). In the following we shall perform all calculations with both the above values of $\theta_{\mathrm{Nb}}$.

\subsection{Choosing the Values of $T_{c}$ for Which the Data in [9] Are Addressed}

In [9], while values of $T_{c}$ varying between 9.87 and $15.25 \mathrm{~K}$ have been reported for 13 samples of $\mathrm{NbN}$ for which the values of $j_{0}$ lie in range $2.92-13.30 \mathrm{MA} \cdot \mathrm{cm}^{-2}$, the values of $n_{e}$ have been reported at only three values of $T_{c}$, which are 10.72, 14.02, and 15.17 K. Hence we limit the scope of this paper to these values of $T_{c}$ only.

\subsection{A Consistency Check of (6)}

If we solve the usual BCS equation for $T_{c}$ (i.e., the equation sans $E_{F}$ ) with $\theta=105.7$ $(397.8 \mathrm{~K})$ and $T_{c}=10.72 \mathrm{~K}$, we obtain $\lambda=0.4142(0.2682)$. These are precisely the values we obtain via (6) for the same values of $T_{c}$ and $\theta$ and the additional input of $\mu$ (or $E_{F}$ ) $=100 k \theta$ for each value of $\theta$ being considered. Note that $\mu / k \theta=100$ manifestly satisfies constraint (1). It is hence seen that (6) incorporating $\mu$ is a valid generalization of the usual equation sans $\mu$, and may therefore be used for arbitrary values of $\mu$. 


\subsection{Fixing Additional Required Inputs}

Having fixed the values of $\theta_{\mathrm{Nb}}$ and $T_{\mathcal{o}}$ we can carry out steps (A) and (B) spelled out in Section 4.1; to carry out step (C) we additionally need the values of $\gamma$ and the cell parameters of different samples of $\mathrm{NbN}$, which are not given in [9]. We fix these by appealing to the data in [13]. A summary of all the inputs required for this study is given in Table 1. Based on the data in [17], this table includes the estimated values of $\Delta_{0}$ at each of the $T_{c} s$ under consideration

\subsection{Results}

For each of the three values of $T_{c}$ and both the values of $\theta_{\mathrm{Nb}}$ noted above, we carried out steps (A)-(C) noted in Section (4.1) for $100 \leq \mu / k \theta_{\mathrm{Nb}} \leq 1$. For the sake of brevity, presented in Table 2 are the results corresponding to $\theta_{\mathrm{Nb}}=105.7 \mathrm{~K}$ for only those values of $\mu / k \theta_{\mathrm{Nb}}$ for which the calculated values of $j_{0}$ are in close agreement with their experimental values noted in Table 1 . In obtaining these results we have assumed that $\theta_{\mathrm{NbN}}$ and hence $\theta_{\mathrm{Nb}}$ does not change significantly with $T_{c}$-as is seen from the data in [13]. Thus, up to this stage, having fixed the value of $\theta_{\mathrm{Nb}}$ as $105.7 \mathrm{~K}$, we have shown that each subset of the $\left\{T_{c}, j_{0}\right\}$ experimental values can be accounted for by a corresponding set of $\{\mu, \lambda\}$ values. Since it is pertinent to ask if we could have achieved similar agreement by adopting a different value of $\theta_{\mathrm{Nb}}$, we observe that (i) (3) and (6) can be employed only for values of $\mu / k \theta \geq 1$-otherwise we run into complex values because of the factor $\sqrt{\xi+\mu}$; (ii) for $\mu$ as any multiple of $k \theta_{\mathrm{Nb}}$, the value of $\lambda$ calculated via either of these equations must be less than 0.5 in order to satisfy the Bogoliubov constraint, and (iii) for any value of $T_{0}, j_{0}$ increases as $\mu$ is increased.

Table 1. Experimental values of $\left\{T_{c}, j_{0}, n_{e}\right\}$ [9], $\left\{\gamma, a_{0}\right\}$ [13], and $\Delta_{0}$ [17] employed for the study of $\mathrm{NbN}$ in this paper.

\begin{tabular}{cccccc}
\hline$T_{c}(\mathrm{~K})$ & $j_{0}\left(\mathrm{MA} \cdot \mathrm{cm}^{-2}\right)$ & $n_{e}\left(10^{23} \mathrm{~cm}^{-3}\right)$ & $\gamma\left(\mathrm{mJ} \cdot \mathrm{K}^{-2} \cdot \mathrm{gat}^{-1}\right)$ & $\begin{array}{c}\text { Cell parameter } \\
a_{0}\left(10^{-8} \mathrm{~cm}\right)\end{array}$ & $\Delta_{0}(\mathrm{meV})$ \\
\hline 10.72 & 3.81 & 2.59 & 2.61 & 4.032 & 2.06 \\
14.02 & 11.49 & 1.26 & 3.20 & 4.297 & 2.38 \\
15.17 & 13.38 & 1.26 & 3.41 & 4.389 & 2.31 \\
\hline
\end{tabular}

Table 2. Results of calculations for $\theta_{\mathrm{Nb}}=105.7 \mathrm{~K}$. The value of $\mu / k \theta_{\mathrm{Nb}}$ against each $T_{c}$ is the one that led-via the values of $E_{P}, \lambda, y$, and $v_{g}$ (the gram-atomic volume of NbN) - to a value of $j_{0}$ in close agreement with its experimental value noted in Table $1 . v_{g}$ was calculated with the input of $a_{0}$ from Table 1 and the atomic masses of the $N_{b}$ and $N$, as in [8].

\begin{tabular}{ccccccccc}
\hline$T_{c}(\mathrm{~K})$ & $\mu / \mathrm{k} \theta_{\mathrm{Nb}}$ & $\mu(\mathrm{meV})$ & $E_{\mathrm{F}}(\mathrm{meV})$ & $\lambda$ & $y$ & $v_{g}\left(\mathrm{~cm}^{3} \cdot \mathrm{gat}^{-1}\right)$ & $j_{0}\left(\mathrm{MA} \cdot \mathrm{cm}^{-2}\right)$ & $\Delta_{0}(\mathrm{meV})$ \\
\hline 10.72 & 1 & 9.11 & 9.19 & 0.4300 & 4.496 & 13.158 & 3.65 & 1.79 \\
14.02 & 4 & 36.4 & 36.5 & 0.4670 & 3.653 & 15.926 & 11.4 & 2.41 \\
15.17 & 4.75 & 43.3 & 43.3 & 0.4845 & 3.418 & 16.971 & 13.6 & 2.64 \\
\hline
\end{tabular}


We now take up the results following from $\theta_{\mathrm{Nb}}=397.8 \mathrm{~K}$. The least permissible value of $\mu$ corresponding to it, i.e., $\mu=k \theta_{\mathrm{Nb}}$, led to $j_{0}=9.39 \mathrm{MA} / \mathrm{cm}^{2}$ for $T_{c}=10.72 \mathrm{~K}$ and $j_{0}=12.3 \mathrm{MA} / \mathrm{cm}^{2}$ for $T_{c}=14.02 \mathrm{~K}$. Since both these $j_{0}$ values are greater than their experimental counterparts, in the light of observation (iii) above, one might attempt to employ lower values of $\mu$-which is ruled out because of (i). In fact the value $105.7 \mathrm{~K}$ seems like the upper limit for $\theta_{\mathrm{Nb}}$ because we had to employ the least value of $\mu$ corresponding to it in order to achieve agreement between the calculated and the experimental values of $j_{0}$ at $T_{c}=10.72 \mathrm{~K}$. As a concrete example in support of this statement, we note that $\theta_{\mathrm{Nb}}=125 \mathrm{~K}$, led via the least permissible value of $\mu$ corresponding to it to the following results:

$$
\lambda=0.4008, E_{F}=10.84 \mathrm{meV}, y=5.244, j_{0}=4.14 \mathrm{MA} / \mathrm{cm}^{2} \text {. }
$$

Since this value of $j_{0}$ exceeds the experimental value, we need to employ a lower value of $\mu$-which is impermissible because we have already employed for it the lowest allowed value.

Our considerations so far have been based on the derived values of $\theta_{\mathrm{Nb}}$ from $\theta_{\mathrm{NbN}}=$ $335 \mathrm{~K}$. In order to find if there is a lower limit on the value of $\theta_{\mathrm{Nb}}$, we now report our findings based on the values of $\theta_{\mathrm{Nb}}$ derived from the lowest value of $\theta_{\mathrm{NbN}}$ that was noted above, i.e., $250 \mathrm{~K}$. This value leads via (34) and (35) to $\theta_{\mathrm{Nb}}=296.8$ ( $\mathrm{Nb}$ as the upper bob) and $\theta_{\mathrm{Nb}}=78.9 \mathrm{~K}(\mathrm{Nb}$ as the lower bob). Since the former of these values exceeds the upper limit noted above, we did not pursue it any further. For the latter value, we obtained for any assumed value of $\mu / k \theta \geq 1$,

$$
\lambda \geq 0.5392\left(T_{c}=14.02\right) \text { and } \lambda \geq 0.5630\left(T_{c}=15.17\right) .
$$

Because both these values of $\lambda$ are in conflict with the Bogoliubov constraint, we conclude that $\theta_{\mathrm{Nb}}$ cannot be as low as $78.9 \mathrm{~K}$. The value closest to it that yields values of $\lambda$ satisfying the Bogoliubov constraint at both the $T_{c} s$ is $\theta_{\mathrm{Nb}}=100 \mathrm{~K}$, for which, e.g., $\lambda=0.4971\left(T_{c}=15.17\right)$.

Above considerations raise the question: Could $\mu / k \theta_{\mathrm{Nb}}<1$ for $\mathrm{NbN}$ ? If so, it would put $\mathrm{NbN}$ in the category of heavy-fermion SCs [18]. Since there is no compelling reason to believe that this may be so, we did not pursue this idea.

Given in Table 3 are the predicted values of various parameters concomitant with

Table 3. With $\theta_{\mathrm{Nb}}=105.7 \mathrm{~K}$, predicted values of various parameters of $\mathrm{NbN}$ that are concomitant with the calculated values of $j_{0}$ given against each $T_{c}$ in Table 2.

\begin{tabular}{cccccc}
\hline$T_{c}$ & $s\left(E_{F}\right)=m *\left(E_{F}\right) / m_{e}$ & $n_{s}\left(E_{F}\right)\left(10^{20} \mathrm{~cm}^{-3}\right)$ & $n_{s}\left(E_{F}\right) / n_{e}\left(10^{-3}\right)$ & $P_{0}(\mathrm{eV})$ & $v_{0}\left(10^{4} \mathrm{~cm} \cdot \mathrm{sec}^{-1}\right)$ \\
\hline 10.72 & 18.2 & 3.11 & 1.20 & 91.13 & 7.35 \\
14.02 & 11.6 & 12.5 & 9.92 & 44.91 & 5.69 \\
15.17 & 10.95 & 14.8 & 14.8 & 42.84 & 5.74 \\
\hline
\end{tabular}

Notes: (i) The equations employed for the calculation of the above parameters have been derived in [8] and are as follows: $s\left(E_{F}\right)=A_{1}\left(\gamma / v_{g}\right)^{2 / 3} E_{F}^{-1 / 3}\left(A_{1} \simeq 3.305 \times 10^{-10} \mathrm{eV}^{-1 / 3} \cdot \mathrm{cm}^{2} \cdot \mathrm{K}^{4 / 3}\right), n_{s}\left(E_{F}\right)=A_{2}\left(\gamma / v_{g}\right) E_{F},\left(A_{2} \simeq 2.729 \times 10^{7} \mathrm{eV}^{-2} \cdot \mathrm{K}^{2}\right)$, $P_{0}\left(E_{F}\right)=A_{3}(\theta / y)\left(\gamma / v_{g}\right)^{1 / 3} E_{F}^{-2 / 3},\left(A_{3} \simeq 1.584 \times 10^{-6} \mathrm{eV}^{4 / 3} \cdot \mathrm{cm} \cdot \mathrm{K}^{-1 / 3}\right)$,

$v_{0}\left(E_{F}\right)=A_{4}(\theta / y)\left(\gamma / v_{g}\right)^{-1 / 3} E_{F}^{-1 / 3},\left(A_{4} \simeq 1.406 \times 10^{8} \mathrm{eV}^{2 / 3} \cdot \mathrm{sec}^{-1} \cdot \mathrm{K}^{-5 / 3}\right)$. (ii) The product $\left[\mathrm{n}_{\mathrm{s}}\left(E_{F}\right)\right.$ e $\left.\mathrm{v}_{0}\right]$ at each $\mathrm{T}_{\mathrm{c}}$ yields the same value for $\mathrm{j}_{0}$ as was calculated via (14) and given in Table 2. (ii) The values of $E_{3}^{\prime}=E_{3} / E_{F}=P_{0}^{2} /\left(8 s m_{e} E_{F}\right)$ are $1.12 \times 10^{-2}, 1.2 \times 10^{-3}$ and $9.5 \times 10^{-4}$ for $T_{c}=10.72,14.02$ and $15.17 \mathrm{~K}$, respectively, which justify the approximation made in obtaining (32). 
the experimental values of $T_{c}$ and $j_{0}$ of $\mathrm{NbN}$ at the three $T_{c} s$. Among these, the values of $\Delta_{0}$ are in reasonably good agreement with their experimental counterparts (see Table 1), considering that the latter are estimated values based on the data of [17].

\section{A Review of the Results Obtained in [8] in View of the Modified Equation for $y$}

For $\mathrm{Sn}$ and $\mathrm{Pb}$, all our earlier results remain unchanged because solution of (32) for these elements yields the same values for $y$ that were obtained via (11). Since the values of $\mu / k \theta$ that were needed for these elements are rather large, 55 for Sn and 108 for $\mathrm{Pb}$, this result was to be expected; it also establishes that (32) is a valid generalization of (11). To bring out the extent to which the solutions of the two equations differ for low values of $\mu / k \theta$, we note that if we erroneously employ (11) for Sn for $\mu / k \theta=1, \theta=195 \mathrm{~K}$ and $\lambda=0.2516$ (these values are consistent with $\Delta_{0}$ of the SC), then we obtain $y=20.083$ [8]; employment of (11) in this case is erroneous because the equation was obtained by assuming that $\mu / k \theta \gg 1$. On the other hand, solution of (32) for this case leads to $y=21.613$.

For each of the high- $T_{c}$ SCs dealt with in [8], there are two $\theta s-$ say, $\theta_{1}$ and $\theta_{2}$ and two $\lambda s$ in the problem. The $E_{F}$-dependent equation for $y$ that we now need to employ is

$$
1=T\left(\lambda_{1}, \Sigma_{1}^{\prime}, r_{1} y\right)+T\left(\lambda_{2}, \Sigma_{2}^{\prime}, r_{2} y\right)
$$

where $T(\cdots, \cdots, \cdots)$ was defined in (32), $\Sigma_{i}^{\prime}=k \theta_{i} / E_{F}, r_{i}=\theta_{i} / \theta(i=1,2), \quad y=k \theta / E_{2}$, $\theta$ being the Debye temperature of the SC, and $E_{2}$ was defined in (13). It is hence seen that $r_{i} y=k \theta_{i} / E_{2}$-as it ought to be. Without the multipliers $r_{1}$ and $r_{2}, y$ would denote $k \theta_{1} / E_{2}$ in the first term on the RHS of (38) and $k \theta_{2} / E_{2}$ in second term, whereas with the multipliers $y$ has the same definition (i.e., $k \theta / E_{2}$ ) for both the terms.

Equation (38) generalizes (32) to the TPEM scenario; because it explicitly contains $E_{F}$ as a variable, it is also a generalized version of equation (30) in [8]. Upon solving (38) with the input of $\theta, \theta_{1}, \theta_{2}, \lambda_{1}, \lambda_{2}$, and $E_{F}$ as given in [8] for any of the high- $T_{c}$ SCs, we obtain the same value for $y$ that we had obtained earlier. Notwithstanding the fact that all our results reported in [8] remain unchanged is fortuitous-for lower values of $\mu / k \theta$ than were required in [8], we ought to employ the more accurate (38) rather than equation (30) in [8].

\section{Discussion}

In connection with fixing $\theta_{\mathrm{Nb}}$, we recall that Debye temperature is just another way to specify Debye frequency; it is not to be confused with thermodynamic temperature. We now note that, based on neutron powder diffraction experiments, different values of Debye temperature for the constituents of anisotropic LCO have been reported [19]. This lends support to the idea that the Debye temperature of a composite SC needs to be "resolved." The results reported here depend only on the value of $\theta_{\mathrm{Nb}}$, for the identification of which we have simply employed (34) and (35) as a vehicle.

Among the five variables that determine $j_{0}$-see Equation (14) $-v_{g}$ seems to stand alone. We draw attention to a discussion of this variable in [8]. 


\section{Conclusions}

The main results of this paper are: (i) a new $E_{F}$-dependent equation for the dimensionless construct $y$ defined in (2) has been derived, (ii) it has been shown that the experimental values of $T_{\mathcal{c}} \quad j_{0}$, and $\Delta_{0}$ of $\mathrm{NbN}$ are explicable in the OPEM scenario by a value of $\theta_{\mathrm{Nb}}$ in the range $100-106 \mathrm{~K}$, (iii) predictions have been made about the values of $m^{*}, n_{s}$ and $v_{0}$ that are concomitant with the $T_{c}$ and $j_{0}$ values of $\mathrm{NbN}$, (iv) the greater the value of the ratio $n_{s} / n_{e}$, the greater is the value of $T_{c}$ and (v) it has been pointed out that we need to employ the new equations for $y$ presented here when $\mu \approx k \theta$.

The work reported here is continuation of an attempt to find via theory tangible clues about raising the $T_{c} s$ of composite SCs. The role of experiment in this quest can hardly be over-emphasized. While huge amounts of such data about hundreds of SCs are now available, we have not come across a single composite SC for which all the relevant parameters identified here, i.e., $\theta, T_{c} \Delta_{0}, j_{0}, m^{*}, v_{0}, n_{e}, n_{s}, \gamma$, and $v_{g}$, have been reported.

We conclude by noting that the derivations of most of the equations employed in this paper and the concepts on which they are based, e.g., multiple Debye temperatures, superpropagator, and the Bogoliubov constraint, can be found at one place in [10].

\section{Acknowledgements}

The author thanks Dr. A. Semenov for kindly responding to his queries concerned with the experimental data reported in [9], and Professor D.C. Mattis for encouragement.

\section{References}

[1] Lee, D. (2012) Nature Physics, 8, 364-365.

[2] Zhang, Y., et al. (2012) Nature Physics, 8, 371-375.

[3] Allan, M.P., et al. (2012) Science, 336, 563-567. https://doi.org/10.1126/science.1218726

[4] Lin, X, Zhu, Z., Fauqué, B. and Behnia, K. (2013) Physical Review X, 3, 021002. https://doi.org/10.1103/PhysRevX.3.021002

[5] Alexandrov, A.S. (2001) Physica C: Superconductivity, 363, 231-236. https://doi.org/10.1016/S0921-4534(01)01095-4

[6] Jarlborg, T. and Bianconi, A. (2013) Physical Review B, 87, 054514. https://doi.org/10.1103/PhysRevB.87.054514

[7] van der Marel, D., van Mechelen, J.L.M. and Mazin, I.I. (2011) Physical Review B, 84, 205111. https://doi.org/10.1103/PhysRevB.84.205111

[8] Malik, G.P. (2016) Journal of Superconductivity and Novel Magnetism, 29, 2755-2764.

[9] Semenov, A., et al. (2009) Physical Review B, 80, 054510. https://doi.org/10.1103/PhysRevB.80.054510

[10] Malik, G.P. (2016) Superconductivity: A New Approach Based on the Bethe-Salpeter Equation in the Mean Field Approach. World Scientific, Singapore. https://doi.org/10.1142/9868

[11] Thouless, D.J. (1960) Perturbation Theory in Statistical Mechanics and the Theory of Superconductivity. Annals of Physics, 10, 553-588. https://doi.org/10.1016/0003-4916(60)90122-6

[12] Nozières, P. and Schmitt-Rink, S. (1985) Bose Condensation in an Attractive Fermion Gas: From Weak to Strong Coupling Superconductivity. Journal of Low Temperature Physics, 
59, 195-211. https://doi.org/10.1007/BF00683774

[13] Roedhammer, P., Gmelin, E., Weber, W. and Remeika, J.P. (1977) Observation of Phonon Anomalies in $\mathrm{NbC}_{\mathrm{x}} \mathrm{N}_{1-\mathrm{x}}$ Alloys. Physical Review B, 15, 711-717. https://doi.org/10.1103/PhysRevB.15.711

[14] Geballe, T.H., et al. (1966) High-Temperature SP-Band Superconductors. Physics, 2, 293310.

[15] Pellan, Y., Dousselin G., Pinel, J. and Sohn, Y.U. (1990) Temperature and Magnetic Field Dependence of NbN Film Resistivity: 3D Weak Localization Effects. Journal of Low Temperature Physics, 78, 63-77. https://doi.org/10.1007/BF00682110

[16] Geibel, C., Rietschel, H., Junod, A., Pelizzone M. and Muller, J. (1985) Electronic Properties, Phonon Densities of States and Superconductivity in $\mathrm{Nb}_{1-\mathrm{x}} \mathrm{V}_{\mathrm{x}} \mathrm{N}$. Journal of Physics F: Metal Physics, 15, 405-416. https://doi.org/10.1088/0305-4608/15/2/014

[17] Antonova, E.A., Dzhuraev, D.R., Motulevich, G.P. and Sukhov, V.A. (1981) Superconducting Energy Gap of Niobium Nitride. Journal of Experimental and Theoretical Physics, 53, 1270-1271.

[18] Malik, G.P. (2015) A Study of Heavy-Fermion Superconductors via BCS Equations Incorporating Chemical Potential. Journal of Modern Physics, 6, 1233-1241. https://doi.org/10.4236/jmp.2015.69128

[19] Kwei, G.H., Lawson, A.C. and Mostoller, M. (1991) Vibrational Properties and Atomic Debye Temperature for $\mathrm{La}_{2} \mathrm{CuO}_{4}$ from Neutron Powder Diffraction. Physica C: Superconductivity, 175, 135-142. https://doi.org/10.1016/0921-4534(91)90244-S

\section{Submit or recommend next manuscript to SCIRP and we will provide best service} for you:

Accepting pre-submission inquiries through Email, Facebook, LinkedIn, Twitter, etc. A wide selection of journals (inclusive of 9 subjects, more than 200 journals)

Providing 24-hour high-quality service

User-friendly online submission system

Fair and swift peer-review system

Efficient typesetting and proofreading procedure

Display of the result of downloads and visits, as well as the number of cited articles

Maximum dissemination of your research work

Submit your manuscript at: http://papersubmission.scirp.org/

Or contact jmp@scirp.org 